\title{
Magnesium Development as a Lightweight Material_-In Competition with Other Structural Materials
}

\author{
Alan A. Luo
}

\begin{abstract}
Magnesium is emerging as a lightweight material for mass reduction and structural efficiency in the automotive, aerospace and consumer industries, but is currently only a niche material competing with other structural materials such as advanced high-strength steels, aluminum and carbon-fiber-reinforced polymers. This talk will discuss what the magnesium community can learn from our competition, what critical technologies needed for magnesium to become a main stream material for automotive lightweighting. This talk will also present successful examples of multi-material lightweighting in the automotive industry, and highlight the role of magnesium in providing lightweight solutions when competing with other structural materials. Opportunities and challenges for automotive applications of magnesium will be discussed.
\end{abstract}

\section{Keywords}

Lightweighting • Magnesium • Transportation applications - Structural materials

The following conclusions will be reached at the end of the presentation:

- Regardless of future energy solutions, lightweighting is a "must" for the automotive and other transportation industries.

- Magnesium can play an important role in multimaterial design and manufacturing of lightweight structures.
- Magnesium can learn from its competition (steel, aluminum and polymer-based composites) in developing new alloys and manufacturing processes.

- Magnesium industry should actively participate in early stages of product development with automotive original equipment manufacturers (OEMs).

- Magnesium community should leverage significant government funding available around the world in developing new technologies and products.

\footnotetext{
A.A. Luo $(\square)$

Department of Materials Science \& Engineering, The Ohio State

University, Columbus, OH 43210, USA

e-mail: luo.445@osu.edu
}

A.A. Luo

Department of Integrated Systems Engineering, The Ohio State

University, Columbus, OH 43210, USA 